\title{
Rifabutin Low Dose-Induced Intermediate Uveitis in a Patient with Acquired Immunodeficiency Syndrome
}

\author{
Agustina C. Palacio ${ }^{1}$, Mercedes Cabrini ${ }^{2}$, Graciela Ben ${ }^{2}$, Emilio M. Dodds ${ }^{1, *}$ \\ ${ }^{1}$ Department of Ophthalmology, Consultores Oftalmológicos, Buenos Aires, Argentina \\ ${ }^{2}$ Department of infectology, Hospital General de Agudos Juan A. Fernandez, Buenos Aires, Argentina \\ *Corresponding author: emdodds@gmail.com
}

\begin{abstract}
Intermediate uveitis is a relatively rare manifestation of Rifabutin induced uveitis. Rifabutin toxicity is unusual at doses of $300 \mathrm{mg}$ daily or less. The purpose of this study is to report the case of an HIV+ patient with unilateral rifabutin induced intermediate uveitis after 4 months of treatment with low dose rifabutin (150 mg daily) in combination with ritonavir and fluconazole. The uveitis resolved after drug discontinuance and the use topical corticosteroids.
\end{abstract}

Keywords: Rifabutin, drug induced uveitis, intermediate uveitis, vitritis, drug interaction

Cite This Article: Agustina C. Palacio, Mercedes Cabrini, Graciela Ben, and Emilio M. Dodds, "Rifabutin Low Dose-Induced Intermediate Uveitis in a Patient with Acquired Immunodeficiency Syndrome.” American Journal of Medical Case Reports, vol. 5, no. 5 (2017): 116-118. doi: 10.12691/ajmcr-5-5-3.

\section{Background}

Rifabutin is a semi-synthetic antimycobacterial from the rifamicin family agent mainly used in the treatment of Mycobacterium avium-complex (MAC) and tuberculosis in AIDS patients. Although anterior uveitis with hypopyon is the most common presentation, numerous cases of rifabutin induced uveitis associated with various degrees of vitritis have been reported previously [1-5]. This side effect seems to be dose dependent and rapidly resolves after topical corticosteroid therapy and rifabutin discontinuation.

We report the case of a rifabutin-induced intermediate uveitis in an HIV+ patient with a low dose of rifabutin.

\section{Case Report}

A 48-year-old white man was referred to the uveitis clinic with decreased vision in his right eye. The patient reported red eye and blurry vision of 4 days of progress. His medical history was significant for HIV infection and pulmonary tuberculosis that had been treated with classic therapy for 2 months and then continued with maintenance therapy for over 4 months. For this condition, he was under highly active antiretroviral combination therapy (HAART) with tenofovir/emtricitabine/atazanavir/ritonavir. He was on isoniazid and rifabutin $150 \mathrm{mg} /$ day as maintenance of the anti-tuberculosis treatment. He was also taking fluconazole $200 \mathrm{mg}$ /week for onychomycosis. His past ocular history was insignificant.

His best-corrected acuity was counting fingers in the right eye and 20/20 in the left eye. His intraocular pressure was $4 \mathrm{mmHg}$ OD and $10 \mathrm{mmHg}$ OS. Anterior segment examination of right eye showed descemet folds, 3+ cells and flare (Figure 1). The right posterior segment examination revealed intense vitritis with snow balls and normal retina (Figure 2). The left eye was unremarkable.

Rifabutin associated uveitis was suspected and his medication was switched from rifabutin to levofloxacin. The patient was started on topical prednisolone acetate $1 \%$ every hour and phenylephrine 5\%-tropicamide $0,5 \%$ every 8 hours.

One week later, his visual acuity improved to 20/100 OD, IOP was $9 \mathrm{mmHg}$, and there was a remarkable reduction in both anterior and posterior segment inflammation. Topical corticosteroids were indicated every 2 hours and dilating drops discontinued. The next week visual acuity of right eye was 20/50, IOP unchanged, anterior chamber showed +0.5 cells, and fundus examination showed less vitreous haze and vitreous condensation inferiorly with snow balls. (Figure 3). Prednisolone acetate $1 \%$ was tapered and discontinued in 15 days.

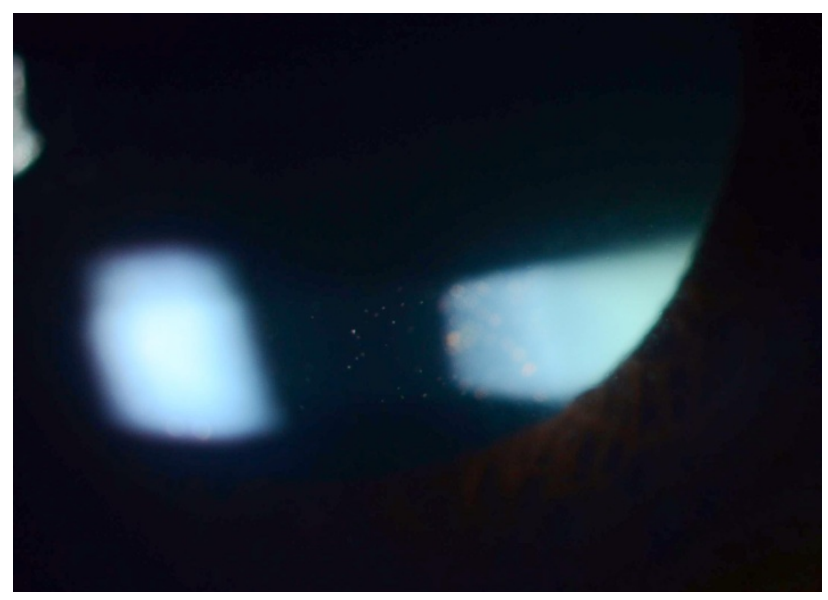

Figure 1. Spillover anterior uveitis 


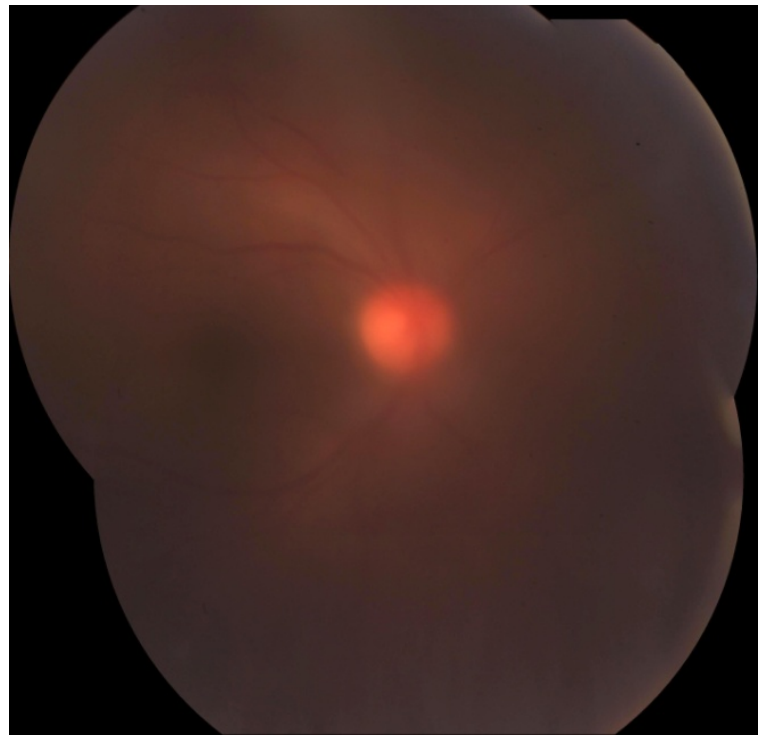

Figure 2. Dense vitritis

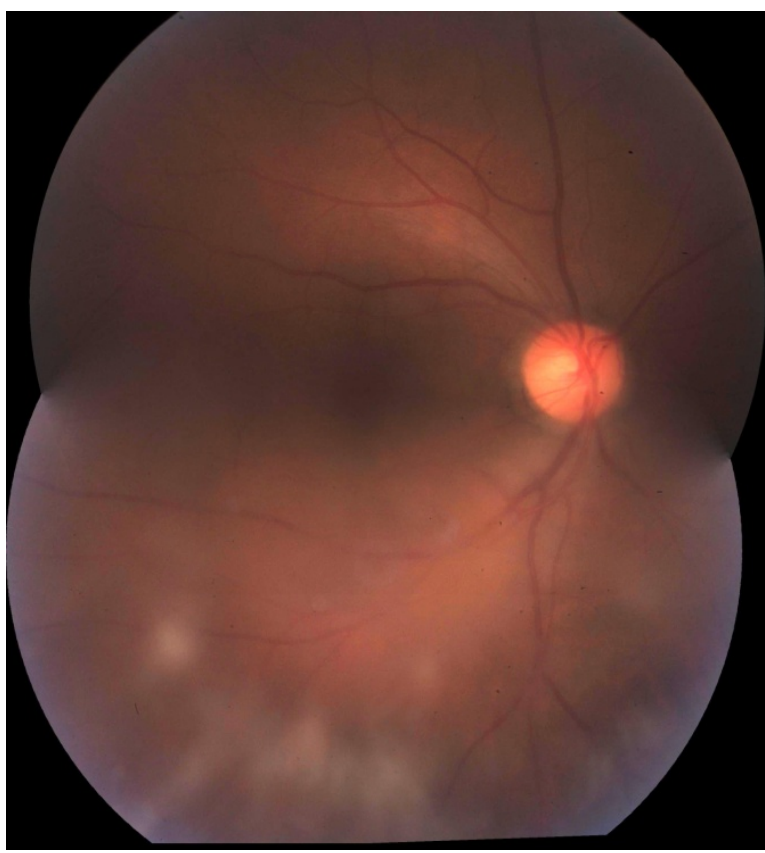

Figure 3. Improvement of intermediate uveitis after 2 weeks' discontinuation of Rifabutin

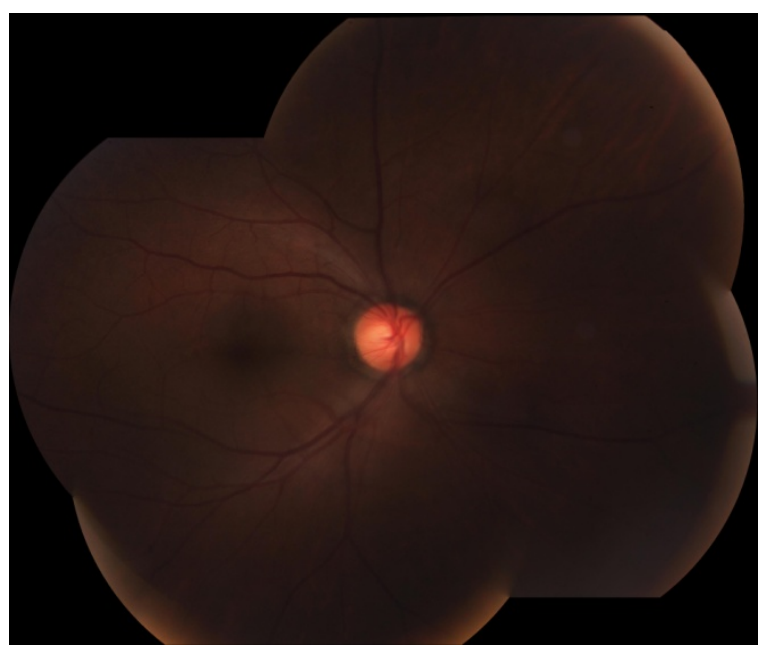

Figure 4. Normal fundus 2 months later after beginning of intermediate uveitis
Two months after the beginning of symptoms, his visual acuity was 20/20 OU and the intraocular pressure 8 $\mathrm{mmHg}$ in both eyes. Anterior and posterior chamber showed a normal exam. (Figure 4).

\section{Discussion}

The most distinctive presentation of rifabutinassociated uveitis is an anterior uveitis with or without hypopyon [1,2,3,4], There are some reported cases however of intermediate uveitis, although this is a relatively infrequent manifestation $[6,7,8]$. Our patient presented with unilateral uveitis which showed a more severe inflammation in the vitreous than in the anterior chamber.

A characteristic of therapy with rifabutin is the dose related toxicity. The dose range of rifabutin associated with uveitis goes between 300 and $1800 \mathrm{mg}$ daily [5,9]. Shafran and colleagues demonstrated that patients on 300 $\mathrm{mg}$ /day of rifabutin rarely developed uveitis, and when it occurred, it took at least 7 months of medication use for the uveitis to develop [3]. Skinner and Blaschke later confirmed that drug related uveitis was unusual at the recommended dose of $300 \mathrm{mg} /$ day [9]. Our patient was on $150 \mathrm{mg}$ daily of rifabutin for 4 months before he developed uveitis but he was also taking ritonavir 100 $\mathrm{mg} / \mathrm{d}$ and fluconazole $200 \mathrm{mg} /$ week. Both drugs interact in the metabolism of rifabutin through inhibition of hepatic cytochrome P450 and may increase the risk of rifabutinassociated uveitis.

Although fluconazole inhibit cytochrome P-450 and increases serum availability of rifabutin [10,11], the association with higher risk of rifabutin induced uveitis is controversial. Shafran and associates found no evidence that concomitant therapy with fluconazole can increase the incidence of uveitis [3]. On the other hand, Havlir et al reported 4 cases of uveitis in patients receiving rifabutin $300 \mathrm{mg}$ daily in combination with fluconazole 400 $\mathrm{mg} /$ week that developed uveitis after 7 months of therapy [12].

Ritonavir is a potent inhibitor of CYP3A and has been demonstrated that it can exacerbate the adverse reactions of rifabutin $[13,14]$. Subsequently, recent guidelines have proposed the use of intermittently administered rifabutin $150 \mathrm{mg} 3$ times per week or $150 \mathrm{mg}$ daily with close monitoring of adverse effects when used in the combination with ritonavir. Investigators found that the serum concentration of rifabutin, when given at dosages of $150 \mathrm{mg}$ every 3 days in combination with ritonavir, were comparable to concentrations achieved with rifabutin 300 mg once daily in patients not being treated with protease inhibitors [15].

There is only one reported case in the literature of an $\mathrm{HIV}+$ cachectic patient with $150 \mathrm{mg} /$ day of rifabutin combined with triple therapy for HIV causing panuveitis and retinal vasculitis after 6 months of treatment [5]. Low body weight is another known risk factor for the development of rifabutin-induced uveitis [3].

We present a well-documented report of an $\mathrm{HIV}+$ non-cachectic patient with unilateral rifabutin induced intermediate uveitis after 4 months of treatment with a low dose of rifabutin (150 $\mathrm{mg}$ qd) in combination with 
ritonavir and fluconazole. The uveitis resolved in 2 months with discontinuation of rifabutin and the use of intensive topical corticosteroids [1,2,4].

\section{Conclusion}

Rifabutin is a well-recognized cause of drug induced intraocular inflammation. Patients receiving rifabutin, even in low doses, with combinations of protease inhibitors and/or azoles should be warned about signs and symptoms of uveitis and be monitored closely for the development of rifabutin toxicity.

\section{Conflict of Interest}

No conflicting relationship exists for any author.

\section{References}

[1] B. R. Saran, A. M. Maguire, C. Nichols, I. Frank, R. W. Hertle, A. J. Brucker, S. Goldman, M. Brown, and B. Van Uitert, "Hypopyon uveitis in patients with acquired immunodeficiency syndrome treated for systemic Mycobacterium avium complex infection with rifabutin," Arch Ophthalmol, vol. 112, no. 9, pp. 1159-65, Sep, 1994.

[2] M. O. Frank, M. B. Graham, and B. Wispelway, "Rifabutin and uveitis,” N Engl J Med, vol. 330, no. 12, pp. 868, Mar 24, 1994.

[3] S. D. Shafran, J. Singer, D. P. Zarowny, J. Deschenes, P. Phillips, F. Turgeon, F. Y. Aoki, E. Toma, M. Miller, R. Duperval, C. Lemieux, and W. F. Schlech, 3rd, "Determinants of rifabutinassociated uveitis in patients treated with rifabutin, clarithromycin, and ethambutol for Mycobacterium avium complex bacteremia: a multivariate analysis. Canadian HIV Trials Network Protocol 010 Study Group,” J Infect Dis, vol. 177, no. 1, pp. 252-5, Jan, 1998.
[4] D. S. Jacobs, P. J. Piliero, M. G. Kuperwaser, J. A. Smith, S. D. Harris, T. P. Flanigan, J. H. Goldberg, and D. V. Ives, "Acute uveitis associated with rifabutin use in patients with human immunodeficiency virus infection," Am J Ophthalmol, vol. 118, no. 6, pp. 716-22, Dec 15, 1994.

[5] S. Skolik, F. Willermain, and L. E. Caspers, "Rifabutin-associated panuveitis with retinal vasculitis in pulmonary tuberculosis," Ocul Immunol Inflamm, vol. 13, no. 6, pp. 483-5, Dec, 2005.

[6] L. Akduman, L. V. Del Priore, H. J. Kaplan, and W. G. Powderly, "Rifabutin induced vitritis in AIDS patients," Ocul Immunol Inflamm, vol. 4, no. 4, pp. 219-24, 1996.

[7] M. J. Chaknis, S. E. Brooks, K. T. Mitchell, and D. M. Marcus, "Inflammatory opacities of the vitreous in rifabutin-associated uveitis,” Am J Ophthalmol, vol. 122, no. 4, pp. 580-2, Oct, 1996.

[8] C. Pochat-Cotilloux, F. De Bats, A. M. Nguyen, F. Benbouzid, A. Malcles, P. Denis, and L. Kodjikian, "[Rifabutin-associated bilateral uveitis: a case report]," J Fr Ophtalmol, vol. 37, no. 8, pp. e115-7, Oct, 2014.

[9] M. H. Skinner, and T. F. Blaschke, "Clinical pharmacokinetics of rifabutin,” Clin Pharmacokinet, vol. 28, no. 2, pp. 115-25, Feb, 1995

[10] P. K. Narang, C. B. Trapnell, J. R. Schoenfelder, J. P. Lavelle, and J. R. Bianchine, "Fluconazole and enhanced effect of rifabutin prophylaxis,” N Engl J Med, vol. 330, no. 18, pp. 1316-7, May 05, 1994.

[11] C. B. Trapnell, P. K. Narang, R. Li, and J. P. Lavelle, "Increased plasma rifabutin levels with concomitant fluconazole therapy in HIV-infected patients,” Ann Intern Med, vol. 124, no. 6, pp. 573-6, Mar 15, 1996.

[12] D. Havlir, F. Torriani, and M. Dube, "Uveitis associated with rifabutin prophylaxis,” Ann Intern Med, vol. 121, no. 7, pp. 510-2, Oct 01, 1994.

[13] A. Cato, 3rd, J. Cavanaugh, H. Shi, A. Hsu, J. Leonard, and R. Granneman, "The effect of multiple doses of ritonavir on the pharmacokinetics of rifabutin," Clin Pharmacol Ther, vol. 63, no. 4, pp. 414-21, Apr, 1998.

[14] H. C. Lin, P. L. Lu, and C. H. Chang, "Uveitis associated with concurrent administration of rifabutin and lopinavir/ritonavir (Kaletra)," Eye (Lond), 12, pp. 1540-1, England, 2007.

[15] J. I. Kuper, and M. D'Aprile, "Drug-Drug interactions of clinical significance in the treatment of patients with Mycobacterium avium complex disease," Clin Pharmacokinet, vol. 39, no. 3, pp. 203-14, Sep, 2000. 